\title{
Pharmacological stress myocardial perfusion imaging after an inadequate exercise stress test
}

\author{
Parija Sharedalal, MD, ${ }^{\text {a }}$ Perry Gerard, $M D{ }^{b}$ and Diwakar Jain, MD, FACC, \\ MASNC $^{\mathrm{C}}$ \\ a Department of Cardiology, Westchester Medical Center, New York Medical College, Valhalla, \\ NY \\ b Department of Radiology, Westchester Medical Center, New York Medical College, Valhalla, \\ NY \\ c Nuclear Cardiovascular Imaging Laboratory, Cardio-Oncology Service, Department of Cardio- \\ vascular Medicine, Westchester Medical Center, New York Medical College, Valhalla, NY
}

Received Mar 20, 2021; accepted Mar 22, 2021

doi: $10.1007 / \mathrm{s} 12350-021-02661-3$

\section{See related article, pp. 1788-1795}

Regadenoson, an adenosine derivative, selectively stimulates $\mathrm{A} 2 \mathrm{a}$ receptors causing increased coronary vasodilation and coronary blood flow, which can be used for pharmacological stress myocardial perfusion imaging (MPI). Regadenoson's selective A2A affinity resulting in fewer undesirable side effects compared to adenosine's $\mathrm{A} 1, \mathrm{~A} 2 \mathrm{~B}$, and $\mathrm{A} 3$ receptor binding has further elevated the drug's benefits over adenosine as proven by the Advance MPI trial. ${ }^{1,2}$ From all the commonly used vasodilators for pharmacological stress MPI: regadenoson, dipyridamole, and adenosine; regadenoson and adenosine are superior in achieving maximum coronary vasodilation. The advanced MPI trial showed regadenoson to be diagnostically non-inferior to adenosine regardless of age, gender, body mass index, and diabetes status. The trial further went on to suggest that regadenoson was very well tolerated overall. FDA approved regadenoson for clinical use in 2008, Currently, this is a widely popular nuclear stress test agent over traditionally used agents.

Exercise and pharmacological stress MPI are important non-invasive modalities for the detection of

Reprint requests: Diwakar Jain, MD, FACC, MASNC, Nuclear Cardiovascular Imaging Laboratory, Cardio-Oncology Service, Department of Cardiovascular Medicine, Westchester Medical Center, New York Medical College, 100 Woods Road, Valhalla, NY 10595; Diwakar.Jain@wmchealth.org

J Nucl Cardiol 2022;29:1796-8.

1071-3581/\$34.00

Copyright (C) 2021 American Society of Nuclear Cardiology. myocardial ischemia in patients with suspected or known coronary artery disease (CAD). ${ }^{3}$ Exercise is the preferred modality for testing in patients who are able to walk on a treadmill. ${ }^{4}$ However, ambulatory patients who are unable to exercise to an adequate workload (inability to achieve $85 \%$ of maximum predicted heart rate and 5 metabolic equivalents or METs), are often converted to pharmacological stress test. Converting a patient to pharmacological stress test after an inadequate treadmill exercise, while using older agents such as adenosine or dipyridamole often results in delay and disruption of the nuclear laboratory's workflow and schedule delays associated with the preparation of weight-based doses and setting up of the infusion pump. Regadenoson is a preferred agent for converting an inadequate exercise stress test to pharmacological stress test given its effortless nature.

Use of regadenoson allows nuclear labs to use it as an add-on agent in patients who undergo exercise stress test but fail to achieve the target heart rate or METs. The paper by Yang et $\mathrm{al}^{5}$ in this issue of the journal demonstrates that regadenoson was the most commonly used agent when patients with inadequate exercise stress test were converted to pharmacological stress test. The use and administration of regadenoson was not considered complex by the nuclear imaging centers' staff and most patients were successfully converted to pharmacologic stress test on the same day in the same session. Unlike adenosine, which is administered as an infusion, regadenoson is administered in a single intravenous bolus of $0.4 \mathrm{mg}$ using a prefilled syringe. ${ }^{6}$ Regadenoson does not require weight-based or creatinine clearancebased dose adjustment, allowing for a seamless transition in select patients from exercise to pharmacological 
stress test. Friedman et al found regadenoson to be the most efficient agent with the shortest total MPI testing time. $^{7}$

The EXERRT trial concluded that administering regadenoson 3 min post-recovery in patients who failed exercise stress test allowed the labs to proceed with pharmacological stress test. ${ }^{8}$ However, caution is required in this regard as to not to proceed with pharmacological stress in patients who develop evidence of ischemia on treadmill exercise at a low workload. Serious adverse events were rare in patients who were converted to pharmacological stress with regadenoson within $3 \mathrm{~min}$ of recovery following an inadequate exercise stress test in this study. Two serious adverse events (unstable angina in one and acute myocardial infarction in one) in a study population of over 1100 patients in EXERRT trial were observed in those patients who developed ST segment depression at a lowlevel exercise, but were nevertheless converted to pharmacological stress within $3 \mathrm{~min}$ of recovery. ${ }^{8}$ One should proceed with injecting the stress dose of radiotracer even at a low work-load in patients who develop evidence of ischemia at a low workload, rather than converting them to pharmacological stress. Ischemia may be easy to detect in those who develop typical symptoms of angina and ischemic ST segment changes. However, it may be sometimes difficult to detect ischemia in the absence of ST segment changes or in the absence of typical anginal symptoms. The stress test operator should remain vigilant about the possibility of ischemia in patients who are unable to achieve adequate workload on treadmill exercise, based upon the clinical history and evaluation of the patient prior to performing stress test. If in doubt about the induction of ischemia on exercise, one should allow adequate recovery period for at least 15-30 min after termination of exercise, before converting the test to pharmacological stress with regadenoson, to avoid developing severe life-threatening ischemia in those with underlying severe CAD. In a previous study comparing pharmacological stress MPI with standard adenosine infusion with adenosine infusion with concurrent low-level treadmill exercise in patients with known or suspected CAD, the later approach was well tolerated and resulted in a reduction of non-cardiac side effects such as flushing and nausea and an improved image quality, but resulted in a greater extent of ischemia in those with CAD. ${ }^{9-11}$

Regadenoson is a well-tolerated and well-liked agent for pharmacologic stress test by the patients. ${ }^{11}$ Overall, regadenoson allows for flexibility and ease in a switch from exercise stress test to pharmacological stress test in a user-friendly and efficient manner compared to other agents in patients who are unable to achieve an adequate workload on treadmill exercise.
However, a rapid switch from treadmill exercise to regadenoson should be limited to only those patients who do not show any evidence of ischemia on treadmill exercise, to avoid the possibility of severe ischemia with superimposed pharmacological stress in these patients.

\section{Disclosures}

Dr Jain serves on the Speakers' Bureau of Astellas. The remaining authors have no conflicts of interest.

\section{References}

1. Cerqueira MD, Nguyen $P$, Staehr P, Underwood SR, Iskandrian AE, Investigators AMT. Effects of age, gender, obesity, and diabetes on the efficacy and safety of the selective A2A agonist regadenoson versus adenosine in myocardial perfusion imaging integrated ADVANCE-MPI trial results. JACC Cardiovasc Imaging. 2008;1:307-16. https://doi.org/10.1016/j.jcmg.2008.02. 003

2. Iskandrian AE, Bateman TM, Belardinelli L, Blackburn B, Cerqueira MD, Hendel RC, et al. Adenosine versus regadenoson comparative evaluation in myocardial perfusion imaging: Results of the ADVANCE phase 3 multicenter international trial. J Nucl Cardiol. 2007;14:645-58. https://doi.org/10.1016/j.nuclcard.2007. 06.114 .

3. Fihn SD, Gardin JM, Abrams J, Berra K, Blankenship JC, Dallas AP, et al. 2012 ACCF/AHA/ACP/AATS/PCNA/SCAI/STS Guideline for the diagnosis and management of patients with stable ischemic heart disease: a report of the American College of Cardiology Foundation/American Heart Association Task Force on Practice Guidelines, and the American College of Physicians, American Association for Thoracic Surgery, Preventive Cardiovascular Nurses Association, Society for Cardiovascular Angiography and Interventions, and Society of Thoracic Surgeons. J Am Coll Cardiol. 2012;60:e44-164. https://doi.org/10.1016/j.ja cc.2012.07.013

4. Gibbons RJ, Balady GJ, Bricker JT, Chaitman BR, Fletcher GF, Froelicher VF, et al. ACC/AHA 2002 guideline update for exercise testing: Summary article. A report of the American College of Cardiology/American Heart Association Task Force on Practice Guidelines (Committee to Update the 1997 Exercise Testing Guidelines). J Am Coll Cardiol. 2002;40:1531-40. https://doi.org/ 10.1016/s0735-1097(02)02164-2.

5. Yang H., Faust, E., Gao, E., Sethi, S., Kitt, T., Kristy, R., Splading, J., Xu, Y. Evaluating the use of pharmacological stress agents during single-photon emission computed tomography myocardial perfusion imaging tests after inadequate exercise stress. American Society of Nuclear Cardiology. 2021.

6. Henzlova MJ, Duvall WL, Einstein AJ, Travin MI, Verberne HJ. ASNC imaging guidelines for SPECT nuclear cardiology procedures: Stress, protocols, and tracers. J Nucl Cardiol. 2016;23:60639. https://doi.org/10.1007/s12350-015-0387-x.

7. Friedman M, Spalding J, Kothari S, Wu Y, Gatt E, Boulanger L. Myocardial perfusion imaging laboratory efficiency with the use of regadenoson compared to adenosine and dipyridamole. Journal of Medical Economics. 2013;16:449-60. https://doi.org/10.3111/ 13696998.2013.772057.

8. Thomas GS, Cullom SJ, Kitt TM, Feaheny KM, Ananthasubramaniam K, Gropler RJ, et al. The EXERRT trial: "EXErcise to 
Regadenoson in Recovery Trial": A phase 3b, open-label, parallel group, randomized, multicenter study to assess regadenoson administration following an inadequate exercise stress test as compared to regadenoson without exercise for myocardial perfusion imaging using a SPECT protocol. J Nucl Cardiol. 2017;24:788-802. https://doi.org/10.1007/s12350-017-0813-3.

9. Thomas GS, Prill NV, Majmundar H, Fabrizi RR, Thomas JJ, Hayashida C, et al. Treadmill exercise during adenosine infusion is safe, results in fewer adverse reactions, and improves myocardial perfusion image quality. J Nucl Cardiol. 2000;7:439-46. https://d oi.org/10.1067/mnc.2000.108030.

10. Thomas GS, Thompson RC, Miyamoto MI, Ip TK, Rice DL, Milikien D, et al. The RegEx trial: A randomized, double-blind, placebo- and active-controlled pilot study combining regadenoson, a selective $\mathrm{A}(2 \mathrm{~A})$ adenosine agonist, with low-level exercise, in patients undergoing myocardial perfusion imaging. J Nucl Cardiol. 2009;16:63-72. https://doi.org/10.1007/s12350-008-9001-9.

11. Kwon DH, Cerqueira MD, Young R, Houghtaling P, Lieber E, Menon V, et al. Lessons from regadenoson and low-level treadmill/regadenoson myocardial perfusion imaging: Initial clinical experience in 1263 patients. J Nucl Cardiol. 2010;17:853-7. h ttps://doi.org/10.1007/s12350-010-9229-z.

Publisher's Note Springer Nature remains neutral with regard to jurisdictional claims in published maps and institutional affiliations. 